\title{
Radiotherapy in marginal zone lymphoma
}

\author{
Kristin Deinbeck', Hans Geinitz¹, Bernhard Haller ${ }^{2}$ and Khashayar Fakhrian ${ }^{1 *}$
}

\begin{abstract}
Purpose: To evaluate the efficacy of radiotherapy (RT) for early-stage nodal and extranodal marginal zone lymphoma (MZL).

Materials and methods: Patients with stage I $(n=22)$ and stage II $(n=8) M Z L$, who were treated with RT were reviewed. The primary tumor localisation was in the orbita $(n=12)$, stomach $(n=8)$, head and neck other than the orbita $(n=8)$, breast $(n=1)$ and one case of marginal zone lymphoma of the skin $(n=1)$. The median radiotherapy dose was 40 Gy (5 to 45 Gy).

Results: The median follow-up time was 103 months. The 5-year overall survival and event-free survival rates were $85 \pm 7 \%$ and $71 \pm 9 \%$, respectively. There was no infield recurrence. Recurrence occurred outside of the radiation field in six patients. The relapses were treated with salvage RT and had excellent local control (100\%) at five years after salvage RT.

Conclusions: Localized extranodal MZL have an excellent prognosis following moderate-dose RT. RT is also an effective salvage therapy in cases of localized recurrence. Further clinical studies should evaluate the optimal dose for MZL.

Keywords: Gastric lymphoma, MALT, Marginal zone lymphoma, Radiotherapy
\end{abstract}

\section{Introduction}

In 1984, Isaacson and Wright [1] described a group of lymphomas that derived from mucosa-associated lymphoid tissue (MALT). Today these lymphomas are well established as a distinct subtype of the marginal zone lymphomas (MZL) in the non-Hodgkin's lymphoma classification. The most common localization is the gastrointestinal tract [2], especially the stomach, but MALT lymphomas arise from other epithelial structures as well. Those include regions in the head and neck area, e.g. the salivary glands, the thyroid gland or the orbita, breast and, though not typically mucosa-associated, in some cases the skin. MZL may also occur primarily in the lymph nodes. The main characteristic of MALT lymphoma is its indolent behaviour. They are commonly diagnosed in localized stages (Ann-Arbor stage I and II) and stay localized for a long time. Previous studies have shown excellent outcomes after radiotherapy in treatment of MALT lymphoma as an organ-preserving treatment modality [3] as has also been shown for other indolent

\footnotetext{
* Correspondence: khfmed@yahoo.com

${ }^{1}$ Department of Radiation Oncology, Klinikum rechts der Isar, Technische Universität München, Munich, Germany

Full list of author information is available at the end of the article
}

lymphoma subtypes [4]. We report the outcomes of a retrospective analysis of 30 patients with stage I-II MALTlymphoma treated with radiotherapy at our Institution.

\section{Methods}

The medical records of 36 patients treated with radiotherapy for Stage I and II MALT lymphoma at our department between 1988 and 2010 were retrospectively reviewed. Inclusion criteria were: histological diagnosis of MALT lymphoma, no transformed MALT lymphoma and no previous treatment. Patients who were treated with chemotherapy previous to radiation $(n=6)$ were excluded. 30 patients fulfilled the inclusion criteria.

Written informed consent was obtained from the patient for publication of this report.

\section{Patient characteristics}

Thirteen men (43\%) and seventeen women (57\%) at a median age of 64 years (range 43-85y) at diagnosis were evaluated for this retrospective analysis.

Twenty-two patients (73\%) presented with MALT lymphoma Ann-Arbor stage I and eight patients (27\%) had lymphoma diagnosed in Ann-Arbor stage II. 
The majority of patients had extranodal disease (90\%). The most common localization in extranodal lymphoma were the orbita (12 patients, $40 \%$ ), stomach (8 patients, $27 \%$ ) and head and neck (5 patients, 17\%). One of the patients with lymphoma of the parotid gland had diagnosed Sjögren's syndrome. One patient (3\%) had a MALT lymphoma of the breast and another patient (3\%) was diagnosed with primary cutaneous lymphoma. Only $10 \%$ of the patients presented with nodal disease. The characteristics of the 30 patients are summarized in Table 1.

\section{Staging}

Upper endoscopy and gastric biopsies were carried out in all the patients with gastric lymphoma, but were not routinely performed in the nongastric cases. H.pylori was documented in biopsy specimens in 2 of the 8 patients with gastric lymphoma. H.p.- eradication therapy prior to RT was unsuccessful Staging included complete blood count, bone marrow biopsy, lactate dehydrogenase (LDH) and appropriate site-specific imaging with CT and/ or MRI in all patients. In addition PET-CT was performed in 2 patients.

\section{Treatment}

RT with 6 - 15 megavoltage photon beams was delivered using a linear accelerator. In one patient with lymphoma of the skin electron beams were used. The median

Table 1 Patient characteristics

\begin{tabular}{ll}
\hline Characteristics & All patients $(\mathbf{n}=\mathbf{3 0})$ \\
\hline Gender & $17(57 \%)$ \\
Female & $13(43 \%)$ \\
Male & \\
Median (range) & 64 years $(43-85$ years) \\
Primary site & \\
Orbital adnexa & $12(40 \%)$ \\
Stomach & $8(27 \%)$ \\
Head and neck & $5(17 \%)$ \\
$\quad$ Parotid gland & $3(60 \%)$ \\
$\quad$ Thyroid & $1(20 \%)$ \\
$\quad$ Other & $1(20 \%)$ \\
Breast & $1(3 \%)$ \\
Cutis & $1(3 \%)$ \\
Nodal & $3(10 \%)$ \\
ECOG & \\
$0-1$ & $27(90 \%)$ \\
2-3 & $2(7 \%)$ \\
Missing & $1(3 \%)$ \\
\hline
\end{tabular}

radiotherapy dose was 40 Gy ( 5 to 45 Gy), delivered at a daily dose of 1.5 to 3.0 Gy. The radiotherapy portals included at least the whole involved organ or nodal region. Eight patients received extended field RT (6 patients with gastric MALT and 2 with nodal MZL). Of the eight patients with gastric MALT lymphoma 6 were treated with abdominal bath and two with irradiation of the whole stomach and the perigastric lymph nodes. Two of the gastric MALT patients had previously been treated with helicobacter-pylory eradication therapy, with no success. Two of the patients with nodal disease received mantle field RT and one involved field RT. Patients with orbital lymphoma received irradiation of the whole orbit without lense shielding. Patients with involvement of the breast, thyroid and parotid gland were treated with RT of the whole organ and adjacent lymph nodes. No patient was treated with chemotherapy.

\section{Follow up}

Patients were clinically examined weekly during RT. Six to eight weeks after the end of the treatment patients were restaged for response and evidence of residual disease. Restaging included a physical examination, relevant radiologic studies, endoscopy in gastric cases and laboratory profiles. The first visit took place $6-8$ weeks after radiation therapy, further follow up was every 3 months for the first year, every 6 months for the next 5 years, and then every year including a physical examination and a site-specific MRI or CT scan. Treatment failure was defined as any kind of recurrence of lymphoma. The first sites of recurrence were recorded as either inside or outside the RT field. Acute side effects were graded according to Common Toxicity Criteria (CTC-3.0) and late sequelae were evaluated using the LENT-SOMA scoring system.

\section{Statistical methods}

Overall Survival (OS) was defined as death from any cause and Event Free Survival (EFS) was defined as the time to relapse or death regardless of cause. OS and EFS rates were estimated using the method of Kaplan and Meier. Log rank tests were performed to compare eventtime distributions between different groups. The results were regarded as significant if $\mathrm{p}$ was $<0.05$.

\section{Results}

Twenty-four patients (80\%) achieved a CR after being treated with RT. In four cases (13\%) a PR was achieved. In two patients $(7 \%)$ response could not specifically be defined, due to death before evaluation and missing data. During a median follow-up time of 103 months, there were 14 events (relapse or death regardless of cause). Ten patients (33\%) died during follow-up. The 5- and 7-year OS was $85 \pm 7 \%$ and $80 \pm 8 \%$, respectively. The 5- and 
7 -year EFS rates were $71 \pm 9 \%$ and $62 \pm 10 \%$, respectively (Figure 1). Stage I disease was significantly associated with a better OS $(\mathrm{p}=0.020)$. The median OS for patients in stage I was not reached and was 104 months (CI 95\% 11-197 months) for patients in stage II, respectively (Figure 2). The 5 and 7-year OS was $94 \pm 6 \%$ and $88 \pm 8 \%$ for patients in stage I and $58 \pm 19 \%$ and $58 \pm 19 \%$ for stage II, respectively. Female gender $(\mathrm{p}=0.137)$ showed a tendency towards better OS. The median OS was not reached for female patients and 104 months (CI 95\% 98-110 months) for male patients, respectively. The 5 and 7 -year OS was $93 \pm 7 \%$ and $85 \pm 10 \%$ for female patients and $85 \pm 10 \%$ and $74 \pm 13 \%$ for male patients, respectively.

No in-field relapses were observed. A total of 6 patients had out-field recurrence (20\%). The median time to recurrence after RT was 26 months (range 8-35 months). Five of the six recurrences occurred after complete remission (CR), one patient had partial remission (PR) after initial treatment: In the first case, recurrence occurred eight months after the end of RT. The patient originally presented with stage I breast MALT lymphoma. She received involved field radiation with 40 Gy and achieved CR. The recurrence occurred in the subcutaneous tissue of the ipsilateral upper arm eight months after primary RT and was treated again with RT. After that, the patient relapsed four more times. Relapses were localized to the head and neck area and the orbita and were treated by RT each time. The median time between the relapses was 24 months. No recurrence was detected 6 months after the last RT.

The second patient had MZL in axillary nodes and relapsed in the parotid gland 24 months after mantle field irradiation. This recurrence was surgically resected. He was free of disease 6 months after the parotidectomy.

The third patient presented with orbital lymphoma and received an involved field RT with 40 Gy. Relapse

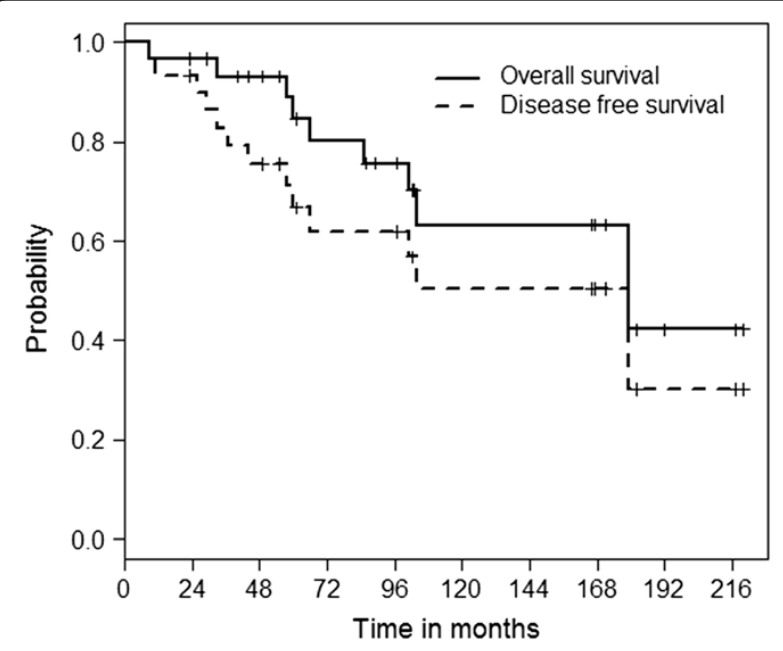

Figure 1 Kaplan-Meier OS and EFS curves of the whole cohort.

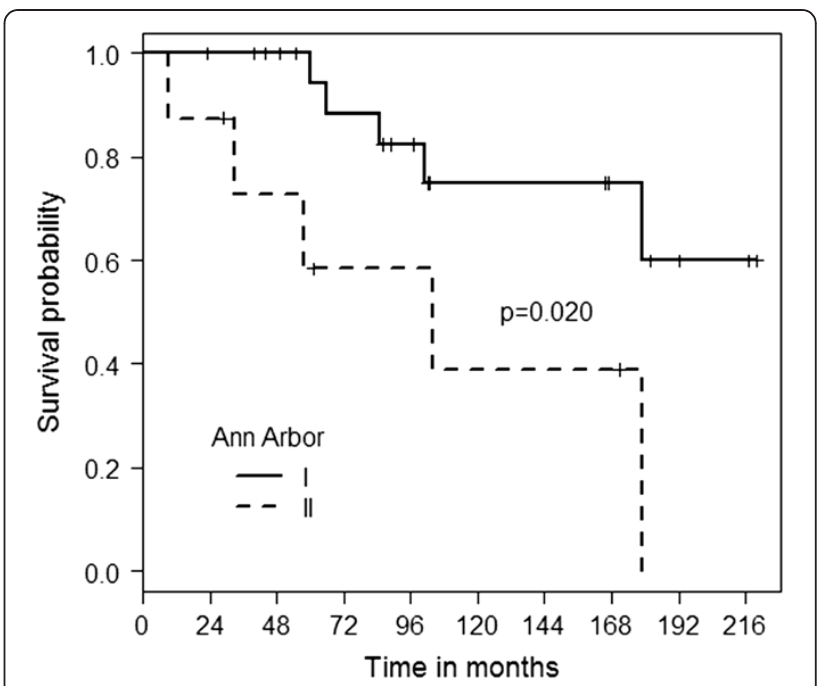

Figure 2 OS for patients with Ann Arbor stage 1 and 2.

was observed in the mucosa of the mouth 27 months after initial treatment and it was treated with 40 Gy involved field RT. A second relapse occurred in the axillary nodes 26 months later and was treated with 50 Gy. This patient was recurrence free for a further 30 months.

The fourth patient presented with primary lymphoma of the orbital adnexa and relapsed after 31 months in a cervical lymph node. Due to age, no further treatment was administered and the patient was alive with disease at the end of follow-up.

The fifth outfield recurrence was also detected in a patient with orbital lymphoma, who was treated with 30 Gy involved field. Thirty-five months after the end of therapy, recurrence occurred in the lung tissue. The recurrence was completely removed by surgery. The patient has been in $\mathrm{CR}$ without new recurrences for 48 months.

The sixth patient received involved field RT with 40 Gy for orbital lymphoma and relapsed after 35 months in the cervical, mediastinal, paraaortal and iliacal lymph nodes. Salvage chemotherapy was administered, but the patient died four months after the diagnosis of recurrence.

\section{Toxicity}

There were no treatment-related deaths. Acute toxicity was seen in patients with lymphoma of the orbita. The most common side effect was $\leq$ grade 3 conjunctivitis (31\%). The most common late toxicity was cataract formation with the need of surgical extraction of the lense in four patients. One patient lost sight after irradiation with 40 Gy Of the eight patients with lymphoma of the stomach, three $(37 \%)$ showed $\leq$ grade 3 acute side effects including diarrhea, nausea and stomach pain. Acute $\leq$ grade 3 mucositis, xerostomia and dysphagia were observed in four cases of head and neck lymphoma. 


\section{Discussion}

We report our single institutional experience in patients with stage I and II nodal and extranodal marginal zone lymphoma who were primarily treated with RT alone at our department. Regarding the distribution of anatomic sites, Thieblemont et al. reported that half of the patients in their study of 108 patients had GI involvement, about $10 \%$ each had disease of the orbit, skin and lung [5]. Goda et al. reported a higher proportion of patients with disease in the orbital adnexa (43\%) than patients with MALT lymphoma of the stomach (15\%). This is in line with our study where most patients had disease of the orbit (40\%), followed by the stomach (27\%) [6]. We observed no in-field recurrence after a median follow-up time of 103 months, which demonstrates that RT-alone is highly effective in achieving local control for localized MALT lymphoma. The 5-year OS rate of $85 \%$ in our cohort is in line with other studies reporting 5 -year OS rates between 87 and 98\% [3,6-11] for stages I and II. For the 5-year LC excellent rates between 95\% and 100\% [3,9-12] have been described. In our study no local relapses were observed after median radiation doses of 40 Gy. However, marginal zone lymphoma tends to show relatively high systemic relapse rates with an emphasis on other mucosal sites. Relapse rates have been reported between 14 and 43\% [6-9,13,14]. Moreover, several authors reported fewer relapses in patients with gastric lymphoma than in other MALT sites. In a study of 70 stage I and II patients, Tsang et al. found that patients with gastric and thyroid lymphoma showed no relapses at all, whereas $22 \%$ of the patients with MALT lymphoma of other origins developed distant recurrences [9]. Similar observations were made by Goda et al. in 192 patients where no patient with gastric MALT lymphoma showed disease progression [6]. In another series of 108 patients, Thieblemont et al. also described a higher recurrence rate for non-GI lymphoma than for patients with involvement of the stomach [5]. For MALT lymphoma of the salivary glands and the orbital adnexa, relapse rates of up to $37 \%$ and $39 \%$, respectively have been reported $[15,16]$. In a series of 17 patients treated with radiation alone for gastric MALT lymphoma, Schechter et al. found an EFS of $100 \%$ after a median follow-up time of 27 months (1168 months) [17]. This is in line with our study, where only patients with lymphoma of the ocular adnexa, breast and primary nodal disease showed relapse whereas patients with gastric lymphoma did not develop any recurrences. Regarding relapse patterns, several authors have described that additional mucosal sites and the other half of a paired organ are more often involved than lymph nodes $[7,11]$. In our study, recurrence occurred in lymph nodes in two cases; three others recurred in mucosal sites including the oral mucosa, the parotid gland and the lung tissue. One patient's recurrence was in the subcutaneous tissue.
Relapsed disease can generally be easily treated by another course of RT $[6,7,9]$. In our study, treatment for relapses was quite heterogeneous. One patient did not receive any further treatment due to age. Two patients were treated surgically and were alive without disease at the time of follow-up. Another patient received salvage chemotherapy but died of lymphoma. Two more patients received another course of RT and both of their lymphomas were controlled locally but further relapses were discovered at other sites. One of those two is still alive and was in remission at the time of follow-up, the other one died due to breast cancer. In summary, administering RT in recurrent disease provides very good local control.

Data for primary nodal MZL is scarce. In a series of 47 patients with primary nodal disease, Tsang et al. found MZL to be associated with worse outcome than in patients with MALT-lymphoma [9]. OS rates up to $79 \%$ with short time to relapse and a failure-free survival of $22 \%$ were described in a series with a relatively high proportion of patients with stage I and II disease (59\%) [18]. The treatment of this entity is still a topic of discussion and includes the use of various chemotherapy regimens as well as the CD20-antibody Rituximab $[19,20]$ In our series, three patients had stage I primary nodal disease at the axillar, supraorbital and cervical lymph nodes, respectively. All three received a total radiation dose of $40 \mathrm{~Gy}$, two of them in form of a mantle field, one as RT of the involved lymph node region. None of the patients received chemotherapy. The patient with primary axillar involvement relapsed after 24 months in the parotid gland, which was treated surgically. All three patients are alive without disease. As we have a limited number of patients, no statement can be made about whether chemotherapy is necessary in patients with low stage primary nodal disease or not, but $\mathrm{RT}$ alone could be considered for these cases. RT doses are still a topic of discussion. Some authors recommend RT doses between 25 and 35 Gy for patients with MALT lymphoma $[6,7,11,21,22]$. For orbital lymphoma, doses between 25 and 30 Gy seem to be sufficient to obtain durable LC and OS [22-24]. In a recent randomized phase III trial by Lowry et al., patients with indolent lymphoma - predominantly follicular and MALT lymphoma - were treated with 40 to $45 \mathrm{~Gy}$ or 24 to $30 \mathrm{~Gy}$, respectively. No difference in response to treatment, LC, EFS and OS was found between the two groups, but a tendency to reduced toxicity could be observed [25]. Contrary to this, Fakhrian et al. investigated stage I-III follicular lymphoma in 50 patients and observed in-field recurrence only in patients who received a RT dose lower than 26 Gy [26]. In the current study only two patients received a radiation dose lower than $30 \mathrm{~Gy}$, thus we cannot draw any conclusions regarding radiation doses lower than $30^{\circ} \mathrm{Gy}$. However, LC and OS were 
similar for patients receiving RT doses of less than or more than 36 Gy in our cohort. Thus, patients in our group had no benefit of higher radiation doses. The use of chemotherapy and the monoclonal CD20-antibody rituximab should be reserved for patients with stage III and IV disease [27].

\section{Conclusion}

RT is a feasible treatment option for early stage MALT lymphoma with excellent LC and OS. The optimal treatment for recurrent disease remains to be defined and mainly depends on the site of relapse (systemic vs. local). Whether or not additional chemotherapy and/or antibody treatment may be beneficial in patients with primary localized nodal disease still remains to be determined by studies with larger patient numbers. RT is also an effective salvage therapy in cases of localized recurrence. Further clinical studies should evaluate the optimal dose for MZL.

\section{Competing interests}

The authors indicated no potential competing interest.

\section{Authors' contributions}

Dr. FH full access to all of the data in the study and takes responsibility for the integrity of the data and the accuracy of the data analysis. All authors read and approved the final manuscript.

\section{Author details}

'Department of Radiation Oncology, Klinikum rechts der Isar, Technische Universität München, Munich, Germany. ${ }^{2}$ Department of Epidemiology and medical statistics, Klinikum rechts der Isar, Technische Universität München, Munich, Germany.

Received: 21 July 2012 Accepted: 15 December 2012 Published: 2 January 2013

\section{References}

1. Isaacson P, Wright DH: Malignant lymphoma of mucosa-associated lymphoid tissue: A distinctive type of B-cell lymphoma. Cancer 1983, 52:1410-1416.

2. Isaacson P: The MALT lymphoma concept updated. Ann Oncol 1995, 6:319-320.

3. Yamashita H, Nakagawa K, Asari T: Radiotherapy for 41 patients with stages I and II MALT lymphoma: a retrospective study. Radiother Oncol 2008, 87:412-417.

4. Heinzelmann F, Engelhard M, Ottinger $\mathrm{H}$, et al: Nodal follicular lymphoma: The role of radiotherapy for stages I and II. Strahlenther Onkol 2010, 186:191-196.

5. Thieblemont C, Bastion Y, Berger F, et al: Mucosa-associated lymphoid tissue gastrointestinal and nongastrointestinal lymphoma behavior: analysis of 108 patients. J Clin Oncol 1997, 15:1624-1630.

6. Goda JS, Gospodarowicz M, Pintilie M, et al: Long-term outcome in localized extranodal mucosa-associated lymphoid tissue lymphomas treated with radiotherapy. Cancer 2010, 116:3815-3824.

7. Hitchcock S, Ng AK, Fisher DC, et al: Treatment outcome of mucosa-associated lymphoid tissue/marginal zone non-Hodgkin's lymphoma. Int J Radiat Oncol Biol Phys 2002, 52:1058-1066.

8. Liao Z, Ha CS, McLaughlin P, Manning JT, et al: Mucosa-associated lymphoid tissue lymphoma with initial supradiaphragmatic presentation: natural history and patterns of disease progression. Int I Radiat Oncol Biol Phys 2000, 48:399-403.

9. Tsang RW, Gospodarowicz MK, Pintilie M, et al: Localized mucosa-associated lymphoid tissue lymphoma treated with radiation therapy has excellent clinical outcome. J Clin Oncol 2003, 21:4157-4164.
10. Tsang RW, Gospodarowicz MK, Pintilie M, et al: Stage I and II MALT lymphoma: results of treatment with radiotherapy. Int I Radiat Oncol Biol Phys 2001, 50:1258-1264.

11. Tomita N, Kodaira T, Tachibana $\mathrm{H}$, et al: Favorable outcomes of radiotherapy for early-stage mucosa-associated lymphoid tissue lymphoma. Radiother Oncol 2009, 90:231-235.

12. Isobe K, Kagami Y, Higuchi K, et al: A multicenter phase II study of local radiation therapy for stage IEA mucosa-associated lymphoid tissue lymphomas: a preliminary report from the Japan Radiation Oncology Group (JAROG). Int J Radiat Oncol Biol Phys 2007, 69:1181-1186.

13. Wenzel C, Fiebiger W, Dieckmann K, et al: Extranodal marginal zone B-cell lymphoma of mucosa-associated lymphoid tissue of the head and neck area: high rate of disease recurrence following local therapy. Cancer 2003, 97:2236-2241

14. Zucca E, Conconi A, Pedrinis E, et al: Nongastric marginal zone B-cell lymphoma of mucosa-associated lymphoid tissue. Blood 2003, 101:2489-2495.

15. Anacak Y, Miller RC, Constantinou N, et al: Primary mucosa-associated lymphoid tissue lymphoma of the salivary glands: a multicenter Rare Cancer Network study. Int J Radiat Oncol Biol Phys 2012, 82:315-320.

16. Bolek TW, Moyses HM, Marcus RB Jr: Radiotherapy in the management of orbital lymphoma. Int J Radiat Oncol Biol Phys 1999, 44:31-36.

17. Schechter NR, Portlock CS, Yahalom J: Treatment of mucosa-associated lymphoid tissue lymphoma of the stomach with radiation alone. J Clin Oncol 1998, 16:1916-1921.

18. Camacho Fl, Algara P, Mollejo M, et al: Nodal marginal zone lymphoma: a heterogeneous tumor: a comprehensive analysis of a series of 27 cases. Am J Surg Pathol 2003, 27:762-771.

19. Berger F, Felman P, Thieblemont C, et al: Non-MALT marginal zone B-cell lymphomas: a description of clinical presentation and outcome in 124 patients. Blood 2000, 95:1950-1956.

20. Haidenberger A, Fromm-Haidenberger S, de Vries A: Feasibility and toxicity of concomitant radio/immunotherapy with MabThera (rituximab ${ }^{\circledR}$ ) for patients with Non-Hodkin's lymphoma results of a prospective phase I/II study. Strahlenther Onkol 2011, 187:300-305.

21. Schechter NR, Yahalom J: Low-grade MALT lymphoma of the stomach: a review of treatment options. Int I Radiat Oncol Biol Phys 2000, 46:1093-1103.

22. Fung CY, Tarbell NJ, Lucarelli MJ: Ocular adnexal lymphoma: clinical behavior of distinct world health organization classification subtypes. Int J Radiat Oncol Biol Phys 2003, 57:1382-1391.

23. Zhou P, Ng AK, Silver B, et al: Radiation therapy for orbital lymphoma. Int J Radiat Oncol Biol Phys 2005, 63:866-871.

24. Bessell EM, Henk JM, Wright JE, Whitelocke RA: Orbital and conjunctival lymphoma treatment and prognosis. Radiother Oncol 1988, 13:237-244.

25. Lowry L, Smith P, Qian W, et al: Reduced dose radiotherapy for local control in non-Hodgkin lymphoma: a randomised phase III trial. Radiother Oncol 2011, 100:86-92.

26. Fakhrian K, Klemm S, Keller U, et al: Radiotherapy in stage I-III follicular non-Hodgkin lymphoma. Strahlenther Onkol 2012, Epub ahead of print.

27. Zucca E, Dreyling M: ESMO guidelines working group. Gastric marginal zone lymphoma of MALT type: ESMO clinical practice guidelines for diagnosis, treatment and follow-up. Ann Oncol 2010, 21(Suppl 5):175-176.

doi:10.1186/1748-717X-8-2

Cite this article as: Deinbeck et al:: Radiotherapy in marginal zone lymphoma. Radiation Oncology 2013 8:2.

\section{Submit your next manuscript to BioMed Central and take full advantage of:}

- Convenient online submission

- Thorough peer review

- No space constraints or color figure charges

- Immediate publication on acceptance

- Inclusion in PubMed, CAS, Scopus and Google Scholar

- Research which is freely available for redistribution 